\title{
A DIRECT CURRENT DIELECTROGRAPH FOR RECORDING THE MOVEMENTS OF THE HEART
}

\author{
BY NORMAN R. JOSEPH 1 \\ (From the Department of Internal Medicine, University of Cincinnati College of \\ Medicine, Cincinnati)
}

(Received for publication March 5, 1943)

Studies of cardiac activity by means of amplifying currents of high frequency have been reported by Atzler and Lehmann (1), and by Rosa (2). Their method depends essentially on the changes produced in an electrical field by variation of the volume of blood in the heart and surrounding vessels during the cardiac cycle. The present communication describes a direct current amplifier designed to record changes in the energy of an electrostatic field, established about the region in which physiological activity is to be studied.

No attempt will be made at present to relate the results obtained to the actual volume changes in a quantitative manner. Records, presented in this paper, of the effect of the activity of the heart on the electrostatic field are to be regarded as qualitative and empirical. It should be pointed out that this represents only one possible application of the method. With some modification, it should be applicable to the study of other organs or to other types of circulatory changes, muscular activity, or other physiological effects.

\section{METHOD}

The circuit which has been employed is shown in Figure 1. Two insulated plates of galvanized iron, about 6 inches square, are placed over the heart, each about an inch from the body. These are connected to $2 \mathrm{~B}$ batteries, placing a difference of potential of approximately 90 volts between the plates. In this manner, an electrical capacitance is set up, which varies during the cardiac cycle, due to the variations of the volume of blood in the field between the 2 plates.

The grid voltage varies as the capacitance changes, and this variation produces corresponding but amplified changes in the plate current, which are then recorded. The procedure in obtaining a record is as follows: the plates are set in position and the circuit is balanced by adjusting the resistances $R_{4}$ and $R_{6}$ until no current flows through the galvanometer used for preliminary balancing.

\footnotetext{
1 Present address: Department of Research and Development, Joseph E. Seagram and Sons, Inc., Louisville, Ky.
}

When this adjustment has been made, the Sanborn cardiette is switched into the circuit, and the photographic records are obtained. During this time, the subject holds his breath, a period of 10 to 15 seconds being sufficient to obtain a representative record.

\section{THEORY}

In order to understand the operation of the circuit described above and the relation of the records obtained to cardiac activity, a simple theoretical model will be considered. Figure 2 represents a liquid dielectric, $\alpha$, and a gas dielectric, $\beta$,

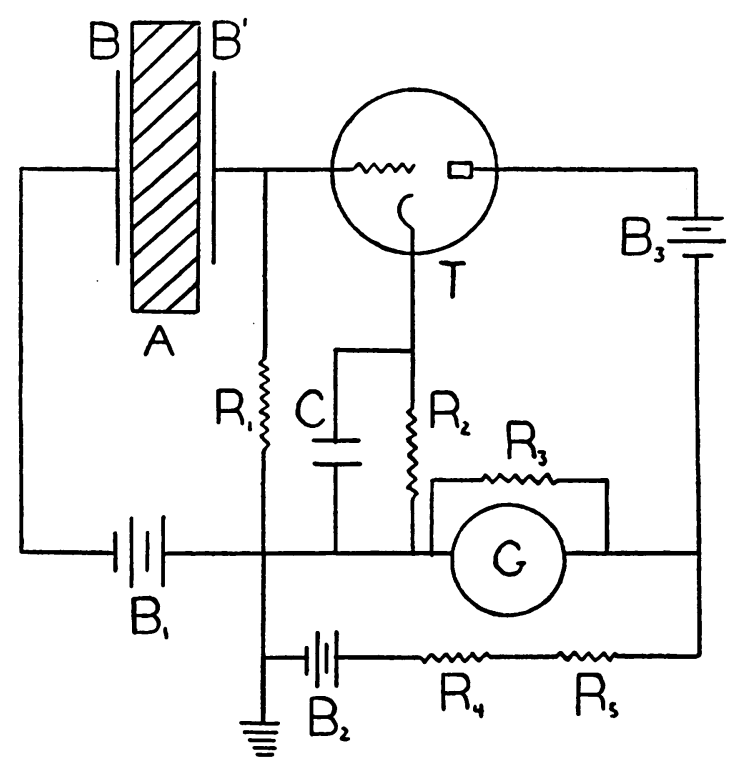

FIG. 1

$A$ is the subject. The condenser plates $B$ and $B^{\prime}$ are placed over the heart, about an inch from the body, and are insulated. The tube $T$ is type $6 \mathrm{C5}$ and is heated with a storage battery. The batteries $B_{1}$ and $B_{3}$ are each of 90 volts, while $B_{2}$ is a 1.5 volt flashlight cell. $R_{1}$ is 2 megohms, $R_{2}$ is 2500 ohms, $R_{3}$ is 1000 to 5000 ohms, $R_{4}$ is 5000 ohms, and $R_{b}$ is 100 ohms. $C$ is 0.5 microfarad. G is a Leeds-Northrup galvanometer used for preliminary balancing, or a Sanborn cardiette for recording. The circuit is shielded by a metal case, which is grounded. The condenser leads are as short as possible and are shielded. 


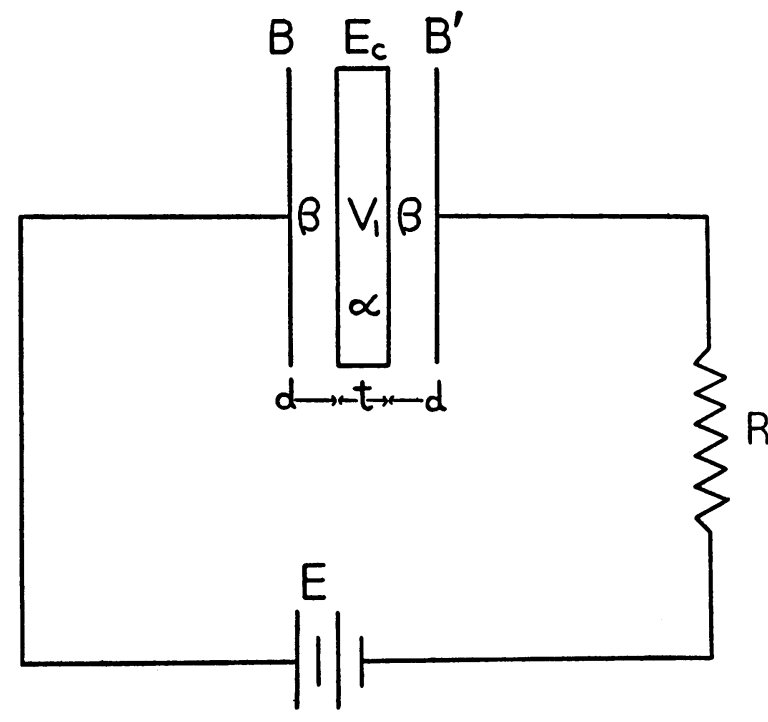

FIG. 2

For explanation see text.

in the electrostatic field of the condenser plates $\mathrm{B}$ and $\mathrm{B}^{\prime}$. The capacitance, $C$, of the condenser is a function of the volume $V_{1}$ of $\alpha$ and the volume $V_{2}$ of $\beta$, and of the dielectric constant of each substance. The total volume between the plates is constant and is determined by the area of the plates and the distance between them.

When the dielectric constant of $\alpha$ is large in comparison with that of $\beta$, as for example in the case of water and air, it is readily shown from elementary electrical theory that

$$
C=C_{0}\left(1+V_{1} / V\right)
$$

where $C$ denotes the capacitance, $C_{0}$ the capacitance when $V_{1}$ is zero, or when the entire space is occupied by $\beta, V_{1}$ is the volume of $\alpha$, and $V$ is the total volume between the plates, a constant.

The capacitance is found to be approximately a linear function of the volume of the liquid dielectric. Reasoning along the same lines for a system of any number of dielectrics in series, the volume of 2 varying in such a manner that their sum is constant, the same conclusion is reached, that the capacitance is a linear function of the variable volume.

This reasoning provides a basis for the provisional assumption that, in intact animals or man, the capacitance change during the cardiac cycle is proportional to the change of volume of the heart. The necessary premises are that the variable parts of the volume are the volume of blood in the heart (high dielectric constant) and the volume of air in the lungs (low dielectric constant). The volumes and configurations of all other structures between the plates are assumed constant.

Having arrived at an approximate relation between the capacitance and the variable volume of the model, it is necessary to consider the relation between capacitance and $E_{c}$, the difference of potential across the condenser plates (Figure 2).

The fundamental equation between $C$ and $E_{0}$ is

$$
E_{c}=q / C
$$

where $q$ is the charge on the plates expressed in coulombs, $C$ the capacitance in farads, and $E_{c}$ the potential difference in volts. When $q$ is maintained constant, $E_{c}$ varies inversely as $C$. In general, one is not justified in regarding $q$ as constant, except for certain limiting cases. Whenever $E_{c}$ changes above or below its equilibrium value $E$, the voltage of the battery, the condenser will tend either to discharge or to be charged, $q$ varying in accordance with the laws of condenser discharge.

From the theory of condenser discharge, three conditions for minimal charging or discharging during any capacitance change can be deduced. First, during an instantaneous change of capacitance, $q$ may be assumed constant, for discharge or charge is a time process. Second, when the resistance $R$ of the circuit is very large, $q$ will tend to remain constant. Third, the tendency to charge or discharge will be minimal when $E_{c}$ approaches $E$.

For each of these cases, the potential difference of the condenser plates varies inversely as the capacitance, which in turn varies linearly with the volume of the heart, according to our hypothesis. Referring to Figure 1, it is evident that the potential of one condenser plate, $B$, is constant. The variable part of the potential is that of the plate $\mathrm{B}^{\prime}$, which is connected directly to the grid of the amplifier tube. The grid voltage determines plate current and the voltage drop across the leads of the recording oscillograph.

Actually, the case of cardiac activity in intact animals or in man must be much more complex than that of any simplified theoretical model which might be postulated, and the relations that have been deduced cannot be assumed to hold in prac- 
tice until many more experimental data have been obtained under a wide range of conditions. In studies on man, one is dealing not only with changes of the heart volume but also with changes of configuration and position. The field between the plates includes not only the heart but also varying sections of the great arteries and veins, and of the pulmonary circulation. In this case, the variable volume is surrounded by a sheath of conducting media, which shields to a large extent the volume and dielectric changes which are to be recorded. These factors vary in every case and would of course be extremely difficult to evaluate in a general manner. Until a large mass of data

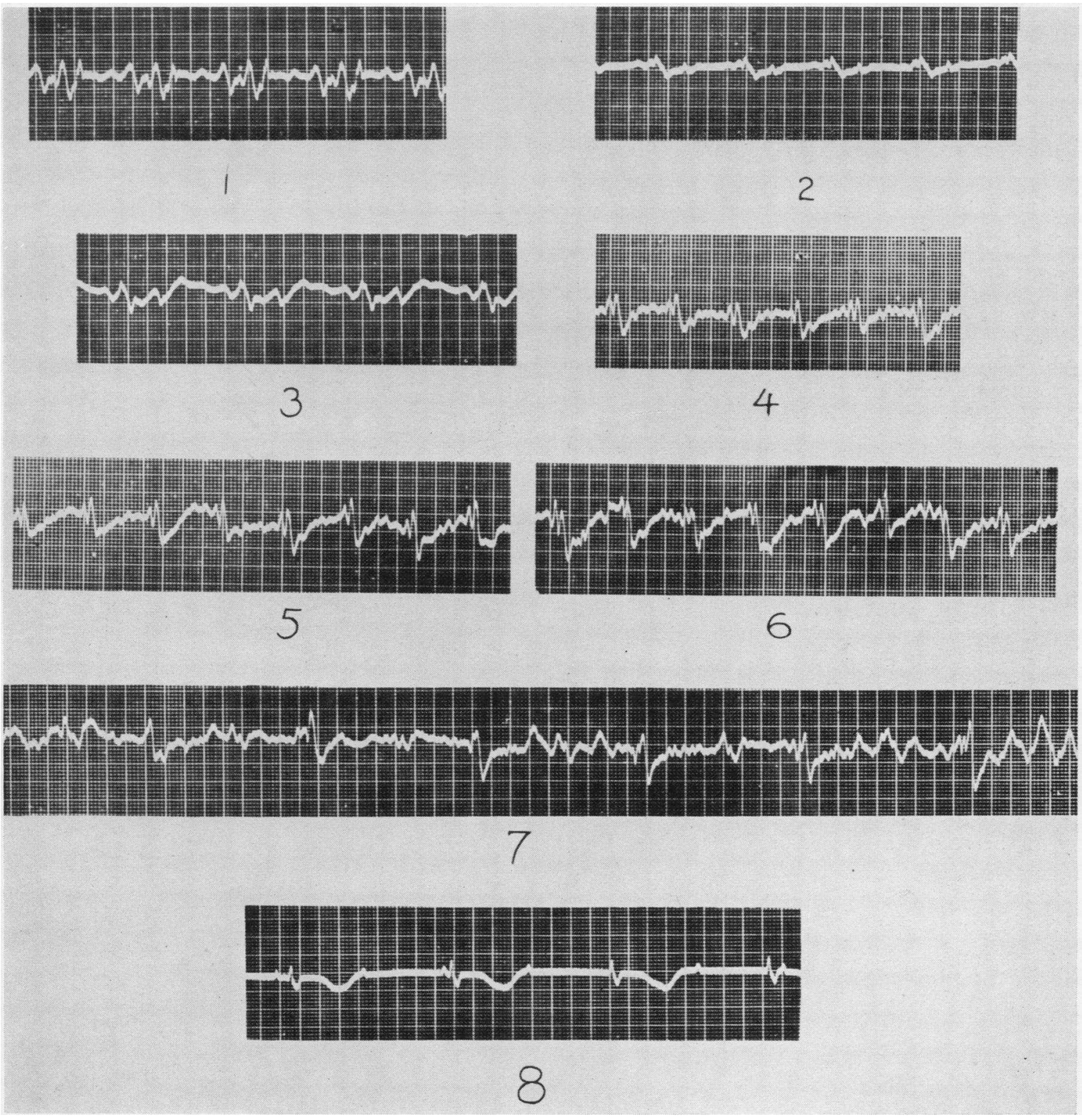

Fig. 3. Dielectrograph Records

1, 2, 3, normal male, aged 22. 4, 5, normal male, aged 30. 6, normal male, age 38. 7, heart block, male hospital patient, age 76. 8, same subject (heart block), electrocardiograph. 
has been studied, a formulation of the relation between capacitance changes and volume changes will not be attempted.

\section{RESULTS}

Records have been obtained on about 20 subjects with normal hearts and on a few subjects with heart disease. Some representative records are presented in Figure 3. These samples include most of the types of record that have been obtained. In many cases, electrocardiographs have been obtained within a few minutes after the condenser cardiograph records were taken. In all of these cases, no significant difference between the heart rates as measured by the two methods could be observed. In one case, the rate was determined by the intervals between the $R$ waves, and in the other case, by the intervals between the major deflections of the condenser cardiograph records. In some cases, electrocardiographs were taken immediately before or after the condenser cardiographs without stopping the camera. In such cases, the $R$ waves were found to correspond in time very closely to the major deflections of the condenser cardiographs, when the time intervals were extrapolated.

Somewhat similar records have been obtained by Atzler and Lehmann (1) and by Rosa (2), who employed a different type of circuit from that described in this communication. They have employed alternating current of radio frequency across the electrodes. The amplifier described in the present paper is much simpler and would seem to be more generally applicable, since it is of a simple direct current type.

No attempt will be made at present to interpret any of the records, except for the statement that, in most of them, the characteristic pattern of each cycle is quite constant, and that, in general, the records tend to be fairly reproducible for a given subject. Obviously, a thorough interpretation would be very difficult because of the many complicating and variable factors that probably affect the results.

One case, however, deserves particular attention. This is the record (Figure 3, No. 7) of a patient with complete heart block. Here, the ventricular rate, about 30 per minute, is found to correspond closely with that of the electrocardiograph (Figure 3, No. 8), and a rhythm of approximately twice this frequency, probably auricular, can be distinguished. Study of a large number of various types of clinical cases might be expected to be of value in interpreting the features of the condenser cardiograph records.

\section{SUM MARY}

1. A direct current amplifier for recording capacitance changes is described, and its application to the study of cardiac activity is indicated.

2. It is shown theoretically that, as first approximations, capacitance varies directly as the dielectric constant and the volume, and that the potential difference across the condenser plates varies inversely with the capacitance.

3. Records of cardiac activity in human subjects are presented and discussed.

4. It is pointed out that, due to a large number of complicating factors, the results can be regarded as having only qualitative and empirical significance.

The author is indebted to Dr. Johnson McGuire for his interest and encouragement.

\section{BIBLIOGRAPHY}

1. Atzler, E., and Lehmann, G., Über ein neues Verfahren zur Darstellung der Herztätigkeit (Dielektrographie). Arbeitsphysiol., 1932, 5, 636.

2. Rosa, L., Diagnostiche Anwendung des Kurzwellenfeldes in der Herz- und Kreislaufpathologie (Radiocardiographie). Ztschr. f. Kreislaufforsch., 1940, 32, 118. 